\title{
Fit of electroweak parameters in polarized deep-inelastic scattering using data from the $\mathrm{H} 1$ experiment
}

\author{
Daniel Britzger*i \\ DESY, Notkestr. 85, 22607 Hamburg, Germany \\ E-mail: daniel.britzger@desy.de
}

\begin{abstract}
The vector and axial-vector couplings of light quarks to the $Z^{0}$-boson are determined using the final deep-inelastic $e^{+} p$ and $e^{-} p$ neutral and charged current scattering cross sections from the H1 experiment at the HERA collider. The fit determines the electroweak parameters together with the parameters of parton distribution functions (PDFs) in order to account for the PDF uncertainties. Compared to an earlier analysis, the precision of the electroweak parameters is improved since the extraction benefits from the data taken with longitudinally polarized electron and positron beams. The work is further extended towards a consistency study of the Standard Model of Particle Physics in the domain of space-like momentum transfer by determining also the masses of the $Z^{0}$ and $W$-bosons, or by determining the Fermi coupling constant $G_{\mathrm{F}}$ or the weak mixing angle $\sin ^{2} \theta_{W}$ together with the $W$-boson mass. The large kinematic reach of the data is exploited and the weak mixing angle, defined in the on-shell scheme, is determined at various scales in the range of the data of $12<Q^{2}<50000 \mathrm{GeV}^{2}$. The results are in agreement with the Standard Model expectations or with other measurements.
\end{abstract}

38th International Conference on High Energy Physics

3-10 August 2016

Chicago, USA

\footnotetext{
*Speaker.

$\dagger$ for the H1 Collaboration and H. Spiesberger
} 


\section{Introduction}

The $\mathrm{H} 1$ experiment at the HERA recorded collisions of electrons (or positrons) of $27.6 \mathrm{GeV}$ and protons of up to $920 \mathrm{GeV}$ and thus provides a large set of cross sections for neutral and charged current deep-inelastic scattering (NC and CC DIS). The data taking periods were subdivided into the HERA-I period during the years 1993 to 2000, and the HERA-II running period in the years 2003 to 2007, where data was taken with an increased luminosity and with longitudinally polarized lepton beams. The inclusive NC and CC cross sections are important ingredients for the determination of parton distribution functions of the proton (PDFs), which are indispensable ingredients for precision measurements and searches for physics beyond the Standard Model (SM) at hadron colliders, such as the LHC. The H1 data on NC and CC scattering has already been combined with data from the ZEUS experiments and the extraction of the PDFs was studied [2].

The H1 inclusive data covers a large kinematic range and also provides cross sections for momentum transfers larger than the $Z$-boson mass with reasonable experimental precision. Therefore, besides the importance for QCD phenomenology, the inclusive DIS data may also be employed for the determination of electroweak parameters of studies of the electroweak Standard Model. At HERA, a first determination of the weak neutral couplings of the light quarks to the $Z^{0}$-boson, the axial-vector coupling, $a_{q}$, and the vector coupling, $v_{q}$, was performed using $\mathrm{H} 1$ data taken during the HERA-I period [3]. These studies are now extended and the full $\mathrm{H} 1$ data for inclusive NC and CC DIS taken during the HERA-I and HERA-II running periods are employed. In particular, the determinations of the weak neutral couplings benefit from data at high values of four-momentum transfers squared $\left(150<Q^{2}<50000 \mathrm{GeV}^{2}\right)$, which were taken during the HERA-II running period with longitudinally polarized lepton beams. Also the increased luminosity reduces significantly statistical uncertainties in an important kinematic range compared to the studies using HERA-I data alone. Recently, the ZEUS Collaboration has reported a similar study using their HERA-II data with longitudinally polarized lepton beams and in addition H1 HERA-I and HERA-II unpolarized cross section measurements [4].

The weak neutral couplings are determined in a combined fit together with parameters of the PDFs in order to account for the uncertainties on the PDFs. The study is extended towards a determination of further parameters of the SM, such as the masses of the $Z$ and $W$-boson, the weak mixing angle $\sin ^{2} \theta_{W}$ or the Fermi coupling constant $G_{\mathrm{F}}$. The precision of these parameters cannot compete with the direct measurements at the $Z$-pole, but the space-like momentum transfer in DIS provides a unique complementary test of the SM

\section{Fit methodology}

The predictions for $\mathrm{NC}$ and $\mathrm{CC}$ cross sections are conveniently expressed in terms of generalized structure functions. For a comprehensive review see for instance reference [5]. The NC DIS interactions are of major importance for the extraction of the weak neutral couplings, and are therefore discussed here in more detail, while, however, also the inclusive CC cross sections are employed and are for instance of importance for the determination of the $W$-boson mass.

$\mathrm{NC}$ interactions in the process $e^{ \pm} p \rightarrow e^{ \pm} X$ are mediated by a virtual photon $(\gamma)$ or $Z^{0}$ boson in the $t$-channel and the cross sections is expressed in terms of generalized structure functions $\tilde{F}_{2}^{ \pm}$, 
$x \tilde{F}_{3}^{ \pm}$and $\tilde{F}_{\mathrm{L}}^{ \pm}$as

$$
\frac{d^{2} \sigma^{\mathrm{NC}}\left(e^{ \pm} p\right)}{d x d Q^{2}}=\frac{2 \pi \alpha^{2}}{x Q^{2}}\left[Y_{+} \tilde{F}_{2}^{ \pm}\left(x, Q^{2}\right) \mp Y_{-} x \tilde{F}_{3}^{ \pm}\left(x, Q^{2}\right)-y^{2} \tilde{F}_{\mathrm{L}}^{ \pm}\left(x, Q^{2}\right)\right]
$$

where $\alpha$ is the fine structure constant. The helicity dependence of the interactions are contained in the terms $Y_{\mp}=1 \pm(1-y)^{2}$ with $y$ being the inelasticity of the process. The generalized structure functions are further expressed separately for pure photon or $Z$-exchange or for the interference of those two as

$$
\begin{aligned}
\tilde{F}_{2}^{ \pm} & =F_{2}-\left(v_{e} \pm P_{e} a_{e}\right) \kappa_{Z} F_{2}^{\gamma Z}+\left[\left(v_{e}^{2}+a_{e}^{2}\right) \pm 2 P_{Z} a_{e}\right] \kappa_{Z}^{2} F_{2}^{Z} \\
x \tilde{F}_{3}^{ \pm} & =-\left(a_{e} \pm P_{e} v_{e}\right) \kappa_{Z} x F_{3}^{\gamma Z}+\left[2 v_{e} a_{e} \pm P_{e}\left(v_{e}^{2}+a_{e}^{2}\right)\right] \kappa_{Z}^{2} F_{3}^{Z},
\end{aligned}
$$

and similarly for $\tilde{F}_{L}$, where the term $\kappa_{Z}$ accounts for $Z$-exchange. It is expressed in the on-shell scheme as

$$
\kappa_{Z}\left(Q^{2}\right)=\frac{Q^{2}}{Q^{2}+m_{Z}^{2}} \frac{G_{\mathrm{F}} m_{Z}^{2}}{2 \sqrt{2} \pi \alpha}
$$

with the Fermi coupling constant calculated from the boson and fermion masses as

$$
G_{\mathrm{F}}=\frac{2 \pi \alpha}{2 \sqrt{2} m_{W}^{2}}\left(1-\frac{m_{W}^{2}}{m_{Z}^{2}}\right)^{-1}(1+\Delta r) .
$$

The term $\Delta r=\Delta r\left(\alpha, m_{W}, m_{Z}, m_{t}, m_{H}, \ldots\right)$ contains corrections to the muon decay and its calculations takes the fine structure constant and the boson and fermion masses as input [6]. The structure functions are related to linear combinations of the quark and anti-quark momentum distributions, $x q$ and $x \bar{q}$, and read for instance for the $F_{2}$ and $x F_{3}$ structure functions in the quark-parton model as:

$$
\begin{aligned}
{\left[F_{2}, F_{2}^{\gamma Z}, F_{2}^{Z}\right] } & =x \sum_{q}\left[e_{q}^{2}, 2 e_{q} v_{q}, v_{q}^{2}+a_{q}^{2}\right]\{q+\bar{q}\}, \\
{\left[F_{3}^{\gamma Z}, F_{3}^{Z}\right] } & =x \sum_{q}\left[2 e_{q} a_{q}, 2 v_{q} a_{q}\right]\{q+\bar{q}\}
\end{aligned}
$$

where $a_{q}$ and $v_{q}$ are the weak neutral vector and axial-vector couplings of the quarks to the $Z$ boson. The couplings are predicted by the SM to be $a_{q}=I_{q, L}^{(3)}$ and $v_{q}=I_{q, L}^{(3)}-2 e_{q} \sin ^{2} \theta_{W}$, with $I_{q, L}^{(3)}$ being the third component of the left-handed isospin. More generally those parameters may be considered as individual parameters to the calculations and thus can be determined explicitly from data. Higher-order weak corrections may be taken into account in terms of form-factors, but these corrections are small and only considered as corrections $\Delta r$ to the muon decay constant $G_{\mathrm{F}}$ and are not considered otherwise.

The neutral couplings of the $u$ and $d$-type quarks $a_{u, d}$ and $v_{u, d}$ are determined in a combined fit together with the parameters of the PDFs. The parameterization and calculations of the PDFs follows closely the methodology as outlined in ref. [2], where we use here calculations in the zeromass variable-flavor-number-scheme (ZM-VFNS) in NNLO accuracy using the program QCDNUM [7] and the data are restricted to $Q^{2} \geq 12 \mathrm{GeV}^{2}$. The goodness of fit quantity, which is 
subject to the Minuit minimization algorithm, is derived from a likelihood function assuming the probability densities to be log-normal distributed:

$$
\chi^{2}=\sum_{i j}\left(\log \left(d_{i}\right)-\log \left(t_{i}\right)\right) V_{i j}^{-1}\left(\log \left(d_{j}\right)-\log \left(t_{j}\right)\right),
$$

where the sum runs over all data points and $d$ and $t$ denote the data and the predictions, respectively, and the covariance matrix $V$ is constructed from all relative uncertainties, taking also correlations between the data sets into account.

\begin{tabular}{l|ccccc}
\hline Dataset & $\begin{array}{c}Q^{2} \text {-range } \\
{\left[\mathrm{GeV}^{2}\right]}\end{array}$ & $\begin{array}{c}\sqrt{s} \\
{[\mathrm{GeV}]}\end{array}$ & $\begin{array}{c}\text { No. of data points } \\
\text { Polarization } \\
{[\%]}\end{array}$ & Ref. \\
\hline$e^{+}$Combined low- $Q^{2}$ & $(0.5) 12-150$ & 319 & $81(262)$ & - & {$[8]$} \\
$e^{+}$Combined low- $E_{p}$ & $(1.5) 12-90$ & 301 & $118(136)$ & - & {$[8]$} \\
$e^{+}$NC 94-97 & $150-30000$ & 301 & 130 & - & {$[9]$} \\
$e^{+}$CC 94-97 & $300-15000$ & 301 & 25 & - & {$[9]$} \\
$e^{-}$NC 98-99 & $150-30000$ & 319 & 126 & - & {$[10]$} \\
$e^{-}$CC 98-99 & $300-15000$ & 319 & 28 & - & {$[10]$} \\
$e^{-}$NC 98-99 high-y & $100-800$ & 319 & 13 & - & {$[11]$} \\
$e^{+}$NC 99-00 & $150-30000$ & 319 & 147 & - & {$[11]$} \\
$e^{+}$CC 99-00 & $300-15000$ & 319 & 28 & - & {$[11]$} \\
$e^{+}$NC L HERA-II & $120-30000$ & 319 & 137 & $-37.0 \pm 1.0$ & {$[12]$} \\
$e^{+}$CC L HERA-II & $300-15000$ & 319 & 28 & $-37.0 \pm 1.0$ & {$[12]$} \\
$e^{+}$NC R HERA-II & $120-30000$ & 319 & 137 & $+32.5 \pm 0.7$ & {$[12]$} \\
$e^{+}$CC R HERA-II & $300-15000$ & 319 & 28 & $+32.5 \pm 0.7$ & {$[12]$} \\
$e^{-}$NC L HERA-II & $120-50000$ & 319 & 138 & $-25.8 \pm 0.7$ & {$[12]$} \\
$e^{-}$CC L HERA-II & $300-30000$ & 319 & 29 & $-25.8 \pm 0.7$ & {$[12]$} \\
$e^{-}$NC R HERA-II & $120-30000$ & 319 & 139 & $+36.0 \pm 0.7$ & {$[12]$} \\
$e^{-}$CC R HERA-II & $300-15000$ & 319 & 28 & $+36.0 \pm 0.7$ & {$[12]$} \\
\hline
\end{tabular}

Table 1: Datasets used in the combined QCD and electroweak fit. The low and medium $Q^{2}$ datasets are combined data samples from all the measurements published by H1 [8]. For each of the datasets, the corresponding range in $Q^{2}$, the center-of-mass energy $\sqrt{s}$, the number of measured data points, and the average polarization value are given. Data taken during the HERA-I running period are taken with unpolarized lepton beams. The numbers in brackets denote the respective quantities for the full dataset, i.e. without the cut of $Q^{2} \geq 12 \mathrm{GeV}^{2}$ applied.

All published $\mathrm{NC}$ and $\mathrm{CC}$ cross sections from $\mathrm{H} 1$ are input to the fit and are summarized in table 1. The cross sections for lower values of $Q^{2}$ at $Q^{2}<150 \mathrm{GeV}^{2}$ have already been combined into a single data set. Cross sections at high- $Q^{2}$ are separately available for the individual running periods, where the data during HERA-II has been taken with longitudinally polarized lepton beams, which improves the sensitivity to $v_{q}$. The beam polarizations are free parameters in the fit and are constrained by taking the measurements of the polarization as additional data points in the calculation of $\chi^{2}$.

\section{Results}

First, the vector and axial-vector couplings of the light quarks, $a_{u, d}$ and $v_{u, d}$ are determined in the QCD and electroweak fitting procedure. The fits are performed using the Alpos fitting 
framework and yield a quality of $\chi^{2} / n_{\mathrm{dof}}=1370.5 /(1388-21)$, where the degrees of freedom is calculated from 1384 cross section data points, 4 polarization values, 13 PDF parameters, 4 quark coupling parameters and 4 polarization values. The results for the weak neutral couplings are displayed in figure 1. The values are found to be in agreement with the Standard Model expec-
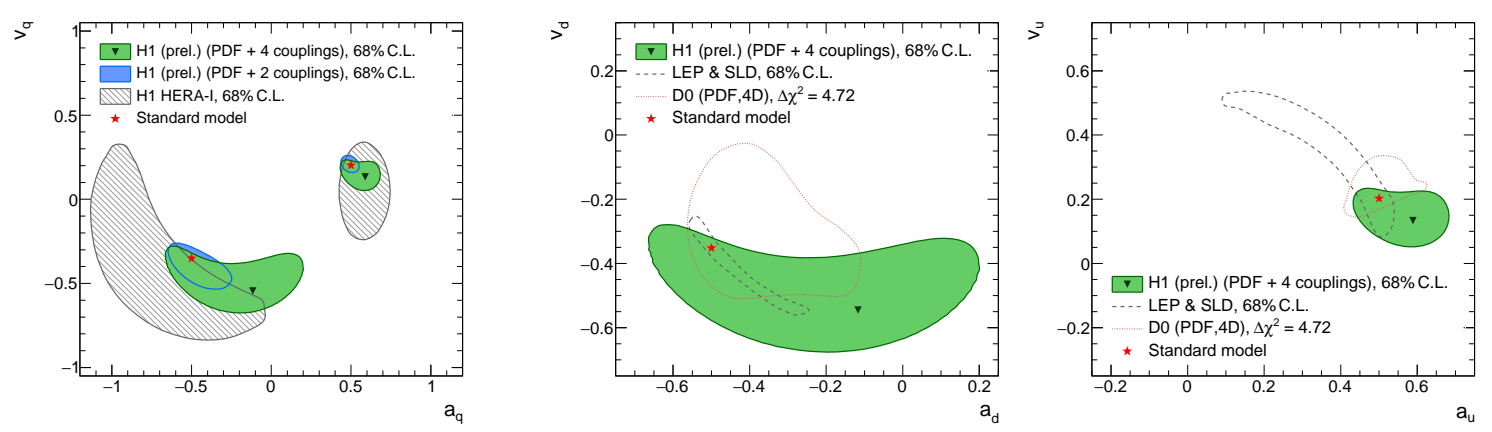

Figure 1: Results for the weak neutral couplings of the $u$ and $d$-type quarks at the $68 \%$ confidence level (C.L.). The results are compared in the left panel to previous results from H1 based on HERA-I data [3] and thus demonstrating the improved precision, which is mainly obtained by including data taken during the HERA-II running period with longitudinally polarized lepton beams. In the middle and right panel the results are compared with results from the LEP experiments and SLD [14], and D0 [15] (the mirror solutions of LEP are not shown). The displayed $68 \%$ C.L. contours of the H1 contours correspond to $\Delta \chi^{2}=2.3$, where at the contour all other fit parameters are minimized.

tations and are within the $68 \%$ confidence interval of the two-dimensional contours of the $u$-type and $d$-type quarks. The precision of the vector couplings has been improved significantly, which is mainly because of the inclusion of the HERA-II data, which was taken with longitudinally polarized lepton beams. When extracting the couplings of the $u$ and $d$-type quarks separately, their uncertainties reduce significantly because the uncertainties on the fit parameters are correlated and the H1 data alone cannot discriminate these correlations. The new results are found to be consistent with extractions from other processes with comparable precision. The different shapes of the contours suggest a large potential to further improve the precision if those different data would be used together for the determination of the weak neutral couplings.

Inclusive NC and CC DIS provides a unique opportunity to test the parameters of the SM in the domain of space-like momentum transfers. The results of the fits of the masses of the $Z$ and $W$-boson, $m_{Z}$ and $m_{W}$, together with the PDFs are displayed in figure 2 . The values are found to be compatible with the best-known values [5] within a confidence level of $68 \%$. Similar fits, where the $Z$-boson mass is substituted and fermi coupling constant, $G_{\mathrm{F}}$, or the weak mixing angle, $\sin ^{2} \theta_{W}$, are fitted together with $m_{W}$ are displayed also in figure 2. For these fits, the electroweak parameters remain defined in the on-shell scheme, and thus the value of $m_{Z}$ is calculated (iteratively) from that parameters together with $m_{W}$ and other boson and fermion masses. The results are compatible with the best-known values of $G_{\mathrm{F}}$ and $\sin ^{2} \theta_{W}$ within a confidence level of $68 \%$.

When taking the value for the $Z$-boson mass $m_{Z}$ as additional external parameter to the fit, the value of $m_{W}$ is determined to

$$
m_{W}=80.407 \pm 0.118(\mathrm{exp}, \mathrm{pdf}) \pm 0.005\left(m_{Z}, m_{t}, m_{H}\right) \mathrm{GeV},
$$



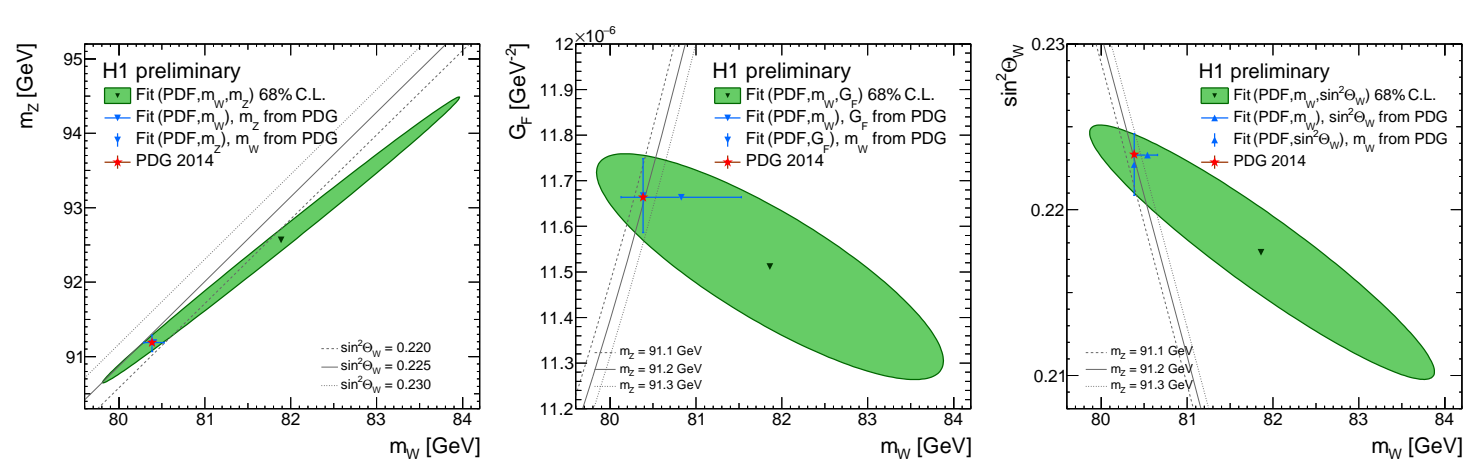

Figure 2: Fits of the PDF parameters and the $W^{ \pm}$-boson mass, together with the $Z^{0}$-boson mass, the Fermi Coupling Constant $G_{F}$ or the Weinberg angle $\sin ^{2} \Theta_{W}$. The respective calculations are all performed in the on-shell scheme, where $\alpha, m_{Z}$ and $m_{W}$ are the only electroweak parameters on Born-level. In case of the latter two fits, the value of $m_{Z}$ is calculated from the fit parameter and $\alpha, m_{W}$ and $\Delta r=\Delta r\left(\alpha, m_{W}, m_{Z}, m_{t}, m_{H}, \ldots\right)$.

where the uncertainty represents the experimental and the PDF uncertainties together. Other uncertainties on $m_{W}$ considered, e.g. the uncertainties of the Z-boson, the top-quark or the Higgs-boson mass are comparably small. Compared to an earlier extraction based on HERA-I data alone [3], the precision is significantly improved, mainly because of the larger dataset of high- $Q^{2}$ cross sections.

The $Q^{2}$-dependence of the inclusive NC and $\mathrm{CC}$ cross sections allows for a determination of the weak mixing angle $\sin ^{2} \theta_{W}$ and a test of its scale dependence. Therefore, the data points for NC and CC DIS are grouped into seven groups with similar or identical values of $Q^{2}$. Each group is assigned an individual value of $\sin ^{2} \theta_{W}$, and a simultaneous fit of all those values of $\sin ^{2} \theta_{W}$ are performed together with the PDF parameters. In this fit $m_{Z}$ is taken as external input parameter, while $m_{W}$ is being calculated. The results for the seven values of $\sin ^{2} \theta_{W}$ at different values of $Q^{2}$ are displayed in figure 3. The extracted values of $\sin ^{2} \theta_{W}$ are consistent over the full $Q^{2}$-range with the best-known value [5]. Despite the uncertainties of the individual values of $\sin ^{2} \theta_{W}$ are correlated, the uncertainties are significantly higher than the one of the well-known value from measurements at the $Z$-pole. However, these values

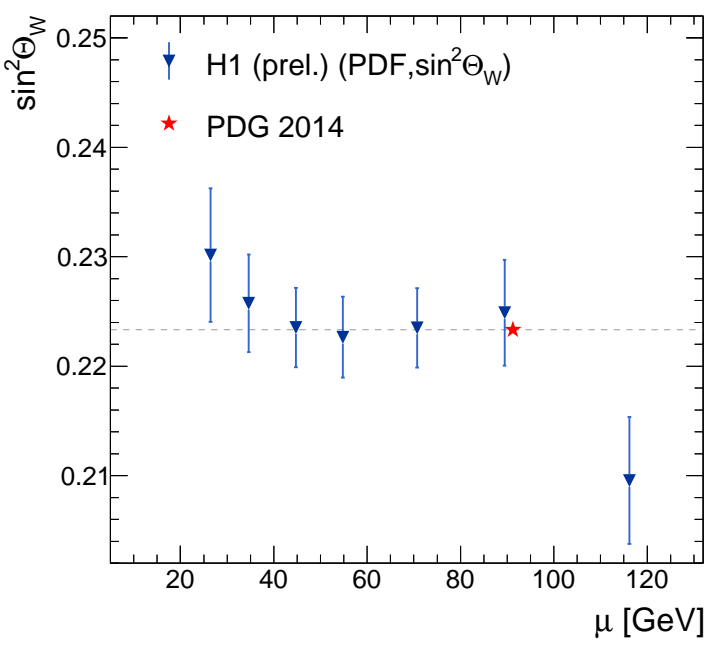

Figure 3: The weak-mixing angle $\sin ^{2} \theta_{W}$ in the onshell scheme definition at different values of $Q^{2}$. The results are compared to the value from measurements at the $Z$-pole.

are valuable data for the scale dependence of $\sin ^{2} \theta_{W}$ at a so far poorly explored scale of $20<\mu<m_{Z}$. 


\section{Summary}

Results from a combined QCD and electroewak fit to all published neutral and charged current scattering data by the $\mathrm{H} 1$ experiment are reported. The usage of HERA-II data taken with polarized lepton beams lead to a substantial improvement of the precision on the weak neutral couplings with respect to the previously published results based on the HERA-I data only. The values of the vector and axial-vector coupling sare found to be compatible with the Standard Model expectations and with determinations from other experiments. The precision of the results are competitive with determinations from other processes.

Beyond the extraction of the quark coupling parameters, also other parameters of the Standard Model, such as the masses of the weak bosons and the weak-mixing angle, are determined and the values are found to be compatible with other measurements. Despite their moderate precisions, these indirect determinations provide a complementary test of the Standard Model compared to the direct measurements at the $Z$-pole.

\section{References}

[1] H1 Collaboration, H1 preliminary report, H1prelim-16-041

[2] H. Abramowicz et al. [H1 and ZEUS Collaborations], Eur. Phys. J. C75 (2015) 12, [arXiv:1506.06042].

[3] A. Aktas et al. [H1 Collaboration], Phys. Lett. B632 (2006) 35, [arXiv:hep-ex/0507080].

[4] H. Abramowicz et al. [ZEUS Collaboration], Phys. Rev. D93 (2016) 092002, [arXiv:1603.09628].

[5] PDG Collaboration, K. A. Olive et al., Chin. Phys. C38 (2014) 090001.

[6] H. Spiesberger, "EPRC: A Program Package for Electroweak Physics at HERA", Contribution to the Workshop "Future Physics at HERA" (1996).

[7] M. Botje, Comput. Phys. Commun. 182 (2011) 490, [arXiv:1005.1481].

[8] F. D. Aaron et al. [H1 Collaboration], Eur. Phys. J. C71 (2011) 1579, [arXiv:1012.4355].

[9] C. Adloff et al. [H1 Collaboration], Eur. Phys. J. C13 (2000) 609, [hep-ex/9908059].

[10] C. Adloff et al. [H1 Collaboration], Eur. Phys. J. C19 (2001) 269, [hep-ex/0012052].

[11] C. Adloff et al. [H1 Collaboration], Eur. Phys. J. C30 (2003) 1, [hep-ex/0304003].

[12] C. Aaron et al. [H1 Collaboration], JHEP 1209 (2012) 061, [arXiv:1206.7007].

[13] V. Andreev et al. [H1 Collaboration], Eur. Phys.J.C75 (2015) 2, [arXiv:1406.4709] .

[14] S. Schael et al. [ALEPH, DELPHI, L3, OPAL and SLD Collaborations], Phys. Rept. 427 (2006) 257, [hep-ex/0509008].

[15] V. M. Abazov et al. [D0 Collaboration], Phys. Rev. D84 (2011) 012007, [arXiv:1104.4590]. 